\title{
Comparaison de la qualité de fromages à pâte pressée cuite fabriqués à partir de lait cru ou microfiltré
}

\author{
Y Bouton 1 , R Grappin 2 \\ 1 Comité interprofessionnel du gruyère de Comté, avenue de la Résistance, \\ BP 26, 39801 Poligny cedex; \\ 2 Station de recherches en technologie et analyses laitières, \\ INRA, BP 89, 39801 Poligny cedex, France
}

(Reçu le 25 mai 1994 ; accepté le 12 octobre 1994)

\begin{abstract}
Résumé - Afin de mettre en évidence le rôle de la flore indigène du lait dans la protéolyse et la flaveur du fromage, des fabrications ont été réalisées en parallèle à partir de lait cru et de lait microfiltré. La protéolyse des fromages a été appréciée à l'aide de plusieurs techniques : mesure des fractions azotées classiques par la méthode de Kjeldahl et des groupements aminés libres par l'acide 2,4,6 trinitrobenzène sulfonique (TNBS), étude par électrophorèse sur gel de polyacrylamide-agarose des caséines et de la fraction azotée soluble par chromatographie liquide haute performance (CLHP). Après 4 mois d'affinage, la composition physico-chimique et la qualité sensorielle des 2 types de fromages sont différentes. En effet, les teneurs en acide acétique et propionique sont plus faibles dans les fromages fabriqués à partir de lait microfiltré, ce qui laisse supposer une élimination de la flore propionique par microfiltration. Ces fromages, en comparaison avec ceux fabriqués au lait cru renferment moins d'acides aminés et petits peptides. Ce sont des produits peu typés et plus amers mais ils sont en revanche moins acides et moins piquants. L'étude montre que les fromages fabriqués au lait cru donnent une meilleure qualité organoleptique globale que les fromages fabriqués au lait microfiltré ; la flore indigène du lait semble donc avoir une influence significative sur la formation de la flaveur.
\end{abstract}

flore indigène / microfiltration / protéolyse / flaveur / minifromage

Summary - Comparison of the final quality of a Swiss-type cheese made from raw or microfiltered milk. The main objectives of this work were to study the influence of the indigenous microflora on the physico-chemical and sensory characteristics of Comté cheese. Experimental mini-cheeses were made from raw and microfiltered milk. To estimate the proteolysis, several analyses were carried out: nitrogen fractions by Kjeldahl method, free amino acids by 2,4,6-trinitrobenzenesulfonic acid (TNBS), agarose-polyacrylamide gel electrophoresis of caseins, high performance liquid chromatography of water soluble extract (HPLC). After 4 months of ripening, the physico-chemical composition and sensory quality of both types of cheeses were different. The acetic and propionic acid levels were lower in 
cheeses made from raw microfiltered milk suggesting that propionic flora was eliminated by microfiltration. These cheeses held less amino acids and small peptides than cheeses made from raw milk. They were also bitter and developed a poor flavor but on the other hand, they were less acid and pungent. The study showed that cheeses made from raw milk gave a better organoleptic quality than cheeses made from microfiltered milk; the indigenous microflora influenced the flavor formation.

indigenous flora / microfiltration / proteolysis / flavor / minicheese

\section{INTRODUCTION}

Le comté est un fromage à pâte cuite pressée, fabriqué à partir de lait cru. Avec une production annuelle d'environ $35000 \mathrm{t}$, il se place au premier rang des fromages d'appellation d'origine contrôlée français (Bertozzi et Panari, 1993). Les levains utilisés sont des cultures de bactéries lactiques thermophiles réalisées soit sur du lactosérum cru, soit sur recuite (lactosérum chauffé à $90^{\circ} \mathrm{C}-95^{\circ} \mathrm{C}$ et acidifié à $\mathrm{pH}$ voisin de 5,50 afin d'éliminer les protéines solubles). Dans une étude précédente (Bouton et al, 1994), nous avons pu montrer que l'influence des bactéries du levain sur la formation d'acides aminés et petits peptides pendant l'affinage se trouvait souvent modifiée par le facteur fromagerie qui regroupe la composition et la qualité bactériologique du lait, la technologie de fabrication et les conditions d'affinage. Nous savons en effet qu'au cours de l'affinage, les bactéries lactiques thermophiles du levain disparaissent tandis que d'autres micro-organismes issus principalement du lait, tels les lactobacilles mésophiles, les pédiocoques ou les bactéries propioniques se développent (Veaux et al, 1974). L'objectif de cette étude était de mettre en évidence le rôle de cette flore secondaire dans la protéolyse et la flaveur d'un fromage à pâte cuite pressée. Dans ce but, nous avons réalisé des fabrications, d'une part à partir de lait cru, et d'autre part à partir de lait épuré par microfiltration tangentielle (Trouvé et al, 1991) afin d'éliminer en grande partie la flore indigène du lait.

\section{MATERIEL ET METHODES}

\section{Souches de bactéries lactiques}

Les bactéries utilisées dans cette étude proviennent d'une collection de souches destinées à la fabrication de comté, pour lesquelles nous disposons d'un ensemble d'informations sur leurs activités protéolytique et acidifiante (Bouton, 1992). Afin de se rapprocher de la technologie comté, nous avons utilisé des levains composés, d'une part de 2 souches de streptocoques thermophiles $\mathrm{S} 18$ et $\mathrm{S} 79$ communes à tous les levains et ayant une activité leucine aminopeptidasique moyenne (respectivement 15,6 et $13,7 \mu \mathrm{g}$ p-nitroaniline/h), et d'autre part de 2 ou 3 souches de lactobacilles choisies en fonction de leur activité protéolytique (Bouton et al, 1993) et de leur origine. Neuf levains ont ainsi été constitués représentant 3 origines géographiques différentes (tableau I). Les mélanges ainsi constitués présentaient tous un $\mathrm{pH}$ voisin de 4,50 après $6 \mathrm{~h}$ d'incubation sur lait à $44^{\circ} \mathrm{C}$.

\section{Fabrications fromagères}

Les fabrications fromagères ont été réalisées selon une technologie páte pressée cuite dans un atelier comprenant 4 minicuves (Bouton et al, 1993). Le lait utilisé chaque jour provenait toujours de la même fromagerie ; à son arrivée, il était écrémé puis microfiltré à l'aide d'un appareil Bactocatch (Alfa-Laval, Les Clayes-sous-Bois, France) d'un débit de $600 \mathrm{l} / \mathrm{h} / \mathrm{m}^{2}$ assurant un facteur de concentration volumique voisin de 20 . L'élimination des bactéries était réalisée "à froid" $\left(35^{\circ} \mathrm{C}\right)$ par microfiltration tangentielle, la taille des pores et la surface de la membrane étant respectivement de $1,4 \mathrm{~mm}$ et $0,2 \mathrm{~m}^{2}$. La crème recueillie était pasteurisée $\left(85^{\circ} \mathrm{C}, 30 \mathrm{~s}\right)$ et réincorporée au lait microfiltré (standardisation en 


\section{Analyses des laits, levains et fromages}

\section{Lait et levains}

Les analyses ont été les suivantes:

- pH mesuré à l'aide d'une électrode de verre Radiometer;

- matière grasse dosée par la méthode Gerber selon la norme NF V04-210 (Anonyme, 1990);

- matière azotée protéique dosée par la méthode colorimétrique au noir amido selon la norme FIL 98A (Anonyme, 1985).

Ces 2 dernières analyses permettent de calculer le urapport fromager", égal au rapport des différences de matière grasse et matière protéique entre le lait et le sérum.

Les analyses microbiologiques ont consisté en la numération de la flore mésophile aérobie du lait cru sur milieu PCA (Plate Count Agar, Difco) après 3 jours d'incubation à $30^{\circ} \mathrm{C}$ (Anonyme, 1991), et de la flore lactique thermophile des laits ensemencés et des levains, sur milieu MRS pour les lactobacilles et sur milieu M17 pour les streptocoques, après 2 jours d'incubation à $42^{\circ} \mathrm{C}$.

\section{Fromages}

Des échantillons de fromages ont été prélevés à $20 \mathrm{~h}$ et à 4 mois en fin d'affinage et analysés selon les méthodes décrites par Bouton et al (1994).

\section{Paramètres physico-chimiques et produits de fermentations}

$\mathrm{pH}$, extrait sec (ES), matière grasse (MG), gras sur $\sec (G / S)$, humiditè du fromage dégraissé (HFD), chlorure de sodium, calcium, isomères $L(+)$ et $D(-)$ du lactate, acides gras volatils.

\section{Protéolyse}

Azote total (NT), azote soluble dans l'eau (NS) après préparation selon une technique adaptée de la méthode de Kuchroo et Fox (1982), azote soluble dans l'azote phosphotungstique (NPT) après préparation selon la technique de Gripon et al (1975), et groupements $-\mathrm{NH}_{2}$ (exprimés en équivalent glycine) dosés sur la fraction NS, par l'acide 2,4,6 trinitrobenzène sulfonique (Bouton et Grappin, 1994) : les fractions NS, NPT et $-\mathrm{NH}_{2}$ sont exprimées en pourcentage par rapport à l'azote total et la fraction NPT est également exprimée en pourcentage par rapport à la fraction NS.

Activité leucine aminopeptidase (Lap, exprimée en $\mu \mathrm{g}$ de $\mathrm{p}$-nitroaniline libéré par $\mathrm{h}$ et par $\mathrm{g}$ de fromage) déterminée sur un broyat de fromage dans l'eau citratée $(2 \mathrm{~g}$ de fromage additionnés de $30 \mathrm{ml}$ d'eau citratée à $2 \%$ sont placés $15 \mathrm{~min}$ à $37^{\circ} \mathrm{C}$, puis homogénéisés pendant 3 min à l'aide d'un ultra-turrax).

Profils chromatographiques par RP-CLHP réalisés sur la fraction NS (chacun des pics est exprimé en unité arbitraire) : l'acquisition et l'intégration des données ont été réalisées à l'aide du logiciel COCONUT (Almanza et Mielle, INRA Dijon, France) ; les graphiques ont ensuite été redessinés dans un format standard à l'aide d'un programme élaboré sous le logiciel SAS (Schlich P, INRA Dijon, France). Pour l'exploitation des résultats, les profils chromatographiques ont été divisés en 10 zones et 6 pics majeurs séparant certaines zones et présents dans tous les chromatogrammes (fig 1).

Profils électrophorétiques des fractions caséines et de leurs produits de dégradation $\left(\alpha_{s 1}\right.$, $\alpha_{\text {s1 -1 }}, \beta$, « $\beta$-dégradée", $\gamma 1, \gamma 2, \beta$ exprimées en pourcentage de l'aire totale des fractions étudiées) ; l'intensité de coloration des bandes d'électrophorèse est suivie à l'aide d'un densitomètre à balayage, Hoefer Scientific Instruments GS300 $(\lambda 580 \mathrm{~nm})$ connecté à un micro-ordinateur muni d'un logiciel de traitement de données spécifique (Bioblock, ref 97568, Illkirch, France).

\section{Évaluation sensorielle}

Elle porte sur l'ensemble des 18 fromages. Les fromages fabriqués le même jour étaient analysés ensemble après 4 mois d'affinage. Cette évaluation sensorielle était de type analytique descriptive, utilisant une échelle de notation à 6 barreaux (intervalles structurés) en vue d'analyses qualitatives et quantitatives ; le jury était formé d'un groupe de 7 personnes entrainées à ce type d'analyse. Les fromages ont été notés (de 0 à 10) sur des critères de pâte (fermeté, élasticité, onctuosité, hétérogénéité) et de goût (intensité, typicité, acide-piquant, amer, salé, mauvais goût). 
Tableau I. Potentiel protéolytique des souches de $L$ helveticus (LH) et de $L$ delbrueckii subsp lactis (LL).

Proteolytic profiles of $\mathrm{L}$ helveticus $(\mathrm{LH})$ and $\mathrm{L}$ delbrueckii subsp lactis (LL).

\begin{tabular}{|c|c|c|c|c|}
\hline \multirow[t]{2}{*}{ Levain } & \multicolumn{2}{|c|}{ Souches } & \multirow[t]{2}{*}{$-\mathrm{NH}_{2}$} & \multirow[t]{2}{*}{ Lap } \\
\hline & $L H$ & $L L$ & & \\
\hline \multirow{3}{*}{ A1 } & 82 & - & 1,30 & 27,6 \\
\hline & 99 & - & 1,63 & 27,9 \\
\hline & 80 & - & 3,07 & 29,0 \\
\hline \multirow[t]{3}{*}{ A2 } & - & 85 & 1,74 & 9,8 \\
\hline & 72 & - & 5,18 & 37,4 \\
\hline & 75 & - & 4,16 & 39,5 \\
\hline \multirow[t]{2}{*}{$A 3$} & - & 90 & 4,07 & 12,7 \\
\hline & 116 & - & 2,05 & 35,9 \\
\hline \multirow[t]{2}{*}{ A4 } & 149 & - & 1,80 & 23,3 \\
\hline & 112 & - & 2,54 & 23,9 \\
\hline \multirow[t]{3}{*}{ A5 } & - & 131 & 1,08 & 7,9 \\
\hline & 122 & - & 1,95 & 27,5 \\
\hline & 124 & - & 1,98 & 24,6 \\
\hline \multirow[t]{2}{*}{ A6 } & - & 139 & 2,72 & 5,5 \\
\hline & 4 & - & 3,53 & 22,5 \\
\hline \multirow[t]{3}{*}{ A7 } & 15 & - & 2,36 & 23,0 \\
\hline & 1 & - & 3,09 & 26,8 \\
\hline & 42 & - & 3,83 & 20,0 \\
\hline \multirow[t]{3}{*}{ A8 } & - & 9 & 1,79 & 11,4 \\
\hline & 54 & - & 3,13 & 27,4 \\
\hline & 57 & - & 2,93 & 40,10 \\
\hline A9 & - & 9 & 1,79 & 11,4 \\
\hline
\end{tabular}

L'activité leucine aminopeptidase (Lap) est exprimée en $\mu \mathrm{g}$ de p-nitroaniline libérée par heure ; les groupements aminés $\left(-\mathrm{NH}_{2}\right)$ sont exprimés en mmol d'équivalent glycine par litre de lait (meq gly/l).

Leucine aminopeptidase activity (Lap) is expressed in $\mu g$ p-nitroaniline/h; free amino acids $\left(-\mathrm{NH}_{2}\right)$ are expressed in mmol equivalent glycine/l milk. matière grasse à $30-31 \mathrm{~g} / \mathrm{l})$. Chacune des associations a fait l'objet de 2 essais conduits en parallèle à partir de lait cru et de lait microfiltré dont les niveaux de flore mésophile aérobie étaient en moyenne respectivement de $1,310^{5}$ et $210^{3}$ $\mathrm{UFC} / \mathrm{ml}$, soit une efficacité de microfiltration voisine de $98 \%$. Les associations $A 2$ et $A 3, A 5$ et $A 6, A 8$ et $A 9$ ont été testées les mêmes jours (tableau II).

La veille de l'expérimentation, les levains étaient préparés au laboratoire sur des milieux utilisés en fromagerie, à partir de cultures mères préparées sur lait écrémé :

- les lactobacilles, ensemencés à $1 \%$ (v/v) sur recuite (additionnée de caillette de veau à $5 \mathrm{~g} / \mathrm{l}$ ), étaient incubés selon un cycle de température: $6 \mathrm{~h}$ à $42^{\circ} \mathrm{C}$ puis descente en $10 \mathrm{~h}$ jusqu'à $25^{\circ} \mathrm{C}$. Au moment de l'utilisation, le $\mathrm{pH}$ de ces cultures atteignait en moyenne 3,50 et la population, 5 $10^{8} \mathrm{UFC} / \mathrm{ml}$;

- les streptocoques thermophiles, ensemencés à $2 \%(\mathrm{v} / \mathrm{v})$ sur lactosérum enrichi à $5 \mathrm{~g} / \mathrm{l}$ de poudre de lait écrémé puis chauffé $1 \mathrm{~min}$ à $75^{\circ} \mathrm{C}$, étaient incubés $6 \mathrm{~h}$ à $42^{\circ} \mathrm{C}$. Au moment de l'emploi, le $\mathrm{pH}$ de ces cultures atteignait généralement 4,60 et la population, $7,2010^{8} \mathrm{UFC} / \mathrm{ml}$.

L'ensemencement en levains thermophiles a été réalisé pendant la maturation $(30 \mathrm{~min}$ ) pour les streptocoques $(0,2 \%)$ et à l'emprésurage pour les lactobacilles $(0,15 \%)$. Une addition complémentaire de présure était réalisée à l'aide de présure à $520 \mathrm{mg}$ de chymosine/l (Granday, Beaune, France).

Tableau II. Plan d'expérience des fabrications fromagères.

Experimental design of cheese making.

\begin{tabular}{|c|c|c|c|c|}
\hline \multirow[b]{2}{*}{$\begin{array}{l}\text { Jour 1: } \\
\text { lait 1 }\end{array}$} & \multicolumn{2}{|c|}{ Cru } & \multicolumn{2}{|c|}{ Microfiltré } \\
\hline & A1 & - & A1 & - \\
\hline $\begin{array}{l}\text { Jour 2: } \\
\text { lait } 2\end{array}$ & $\mathrm{~A} 2$ & A3 & $\mathrm{A} 2$ & $\mathrm{~A} 3$ \\
\hline $\begin{array}{l}\text { Jour } 3 \text { : } \\
\text { lait } 3\end{array}$ & A4 & - & A4 & - \\
\hline $\begin{array}{l}\text { Jour } 4 \text { : } \\
\text { lait } 4\end{array}$ & A5 & A6 & A5 & A6 \\
\hline $\begin{array}{l}\text { Jour 5: } \\
\text { lait } 5\end{array}$ & A7 & - & A7 & \\
\hline $\begin{array}{l}\text { Jour 6: } \\
\text { lait } 6\end{array}$ & A8 & A9 & A8 & A9 \\
\hline
\end{tabular}


Au vu de ces résultats, chaque dégustateur a attribué pour chaque fromage, une note globale de goût et de pâte.

\section{Traitements des résultats}

Une exploitation statistique a été réalisée à l'aide du logiciel Stat-ITCF (version 5, 1991) de l'Institut technique des céréales et des fourrages. Une analyse de variance a permis d'étudier l'influence du facteur lait sur la composition physico-chimique des fromages. Le test des rangs (Wilcoxon, 1945) a été utilisé pour les analyses sensorielles ; ce test tient compte non seulement des signes des différences observées, mais aussi de leurs rangs. Une analyse en composante principale (ACP) a permis de mettre en évidence les relations existant entre les analyses physico-chimiques et les analyses sensorielles.

\section{RÉSULTATS}

\section{Composition des laits et des fromages de $20 \mathrm{~h}$}

Les analyses bactériologiques n'ont pas montré de différence significative de population de flore lactique entre les laits de fabri-

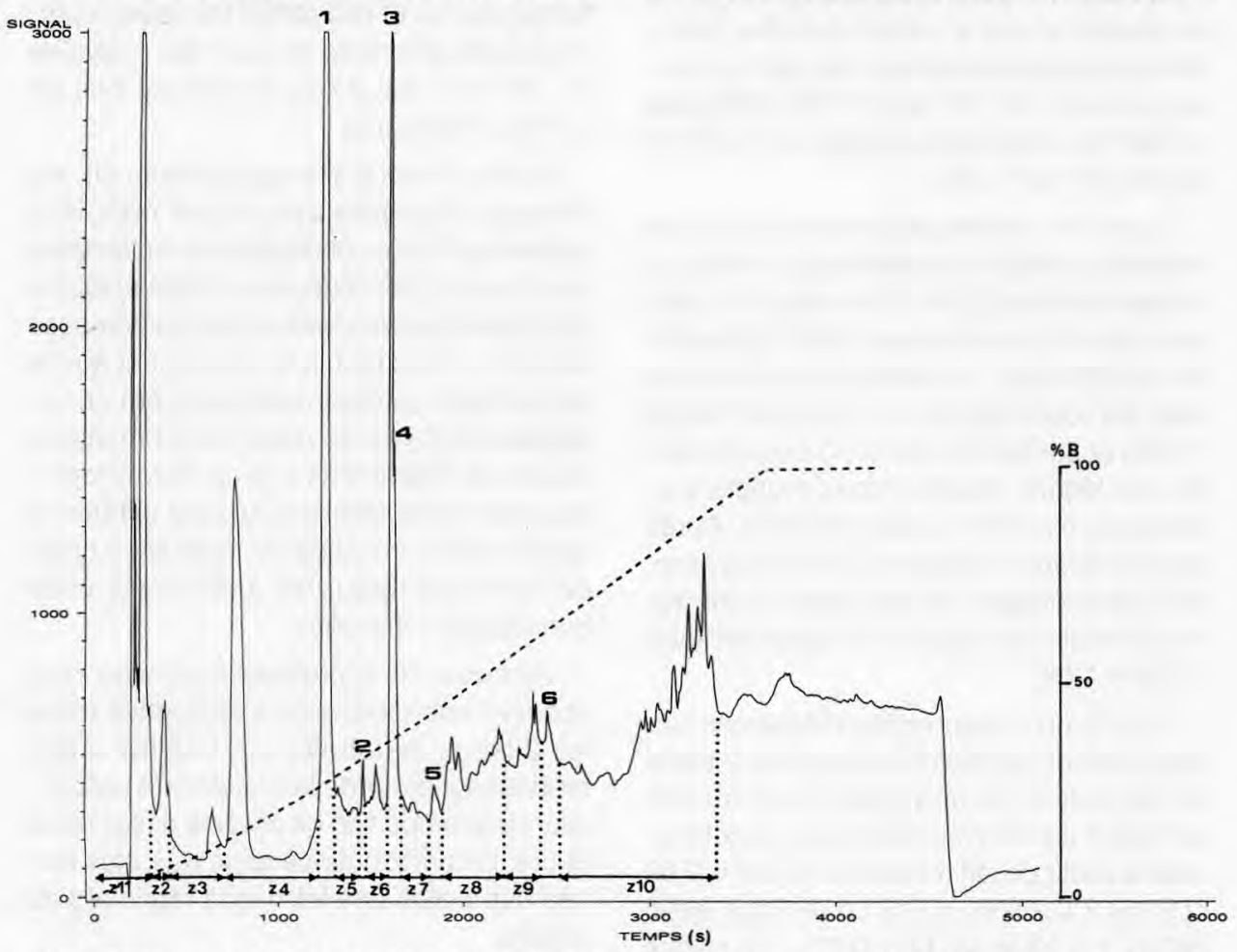

Fig 1. Exemple de profil CLHP de la fraction soluble dans l'eau du comté. Les conditions chromatographiques sont les suivantes : colonne $C 18(4,6 \times 225 \mathrm{~mm})$; solvant $A: 0,1 \%$ d'acide trifluoroacétique dans l'eau; solvant B : $0,1 \%$ d'acide trifluoroacétique dans un mélange d'acétonitrile et d'eau (60/40) ; débit : $0,8 \mathrm{ml} / \mathrm{min}$; détecteur UV $(\lambda=214 \mathrm{~nm})$ et température $30^{\circ} \mathrm{C}$.

Example of HPLC profile of the water-soluble fraction of Comté. Chromatographic conditions were as follows. $C 18$ reverse phase column $(4.6 \times 225 \mathrm{~mm})$; solvant $A, 0.1 \%$ trifluoroacetic acid in water; solvant $B, 0.1 \%$ trifluoroacetic acid in acetonitrile- $\mathrm{H}_{2} \mathrm{O}(60: 40)$; flow rate, $0.8 \mathrm{ml} / \mathrm{min}$; detector UV $\lambda=214$ $\mathrm{nm}$ ) and temperature, $30^{\circ} \mathrm{C}$. 
cation (cru et microfiltré) après ensemencement en levains ; les différences observées étant toutes inférieures à un log. Pour les streptocoques, l'écart entre la population la plus forte observée le $6^{e}$ jour de fabrication $\left(1,5610^{6} \mathrm{UFC} / \mathrm{ml}\right)$ et la population la plus faible observée le premier jour $(1,00$ $10^{6} \mathrm{UFC} / \mathrm{ml}$ ) était d'environ $0,2 \mathrm{log}$; pour les lactobacilles, l'écart entre la population moyenne la plus forte $\left(1,3710^{6} \mathrm{UFC} / \mathrm{ml}\right.$, avec le levain $A 6$ ) et la population moyenne la plus faible $\left(0,1810^{6} \mathrm{UFC} / \mathrm{ml}\right.$, avec le levain A1) était également inférieur à 1 log. Par ailleurs, aucune différence significative n'est ressortie entre la population moyenne de streptocoques et de lactobacilles mesurée après ensemencement sur lait cru (respectivement $1,4110^{6}$ et $0,6710^{6} \mathrm{UFC} / \mathrm{ml}$ ) et sur lait microfiltré (respectivement 1,32 $10^{6}$ et $0,6410^{6} \mathrm{UFC} / \mathrm{ml}$ ).

Le lait microfiltré présentait un taux de matière protéique plus faible que le lait cru (respectivement $27,44 \mathrm{~g} / \mathrm{l}$ contre 29,31 ) lié à une perte de protéines pendant l'opération de microfiltration. Ce résultat était en accord avec les observations de Vincens et Tabard (1988) et Jaubert et al (1991) qui ont montré une légère rétention de la matière protéique au cours de la microfiltration. Après standardisation du lait en matière grasse, les 2 technologies (lait microfiltré et lait cru) ont conduit à un rapport fromager similaire $(1,03$ et 1,06$)$.

À $20 \mathrm{~h}$, on a observé des différences statistiquement significatives entre les 2 types de fabrication, les fromages au lait cru présentaient un ES plus faible que ceux fabriqués à partir de lait microfiltré $(61,88 \pm 0,69$ et $62,54 \pm 0,29$ ) et un $\mathrm{G} / \mathrm{S}$ un peu plus élevé $(47,47 \pm 0,13$ et $47,13 \pm 0,41)$. L'humidité dans le fromage dégraissé était un peu plus importante dans les fromages au lait cru $(53,97 \pm 0,67$ et $53,12 \pm 0,18)$; toutefois, compte tenu des écart types, les différences de composition physico-chimique entre les 2 types de fabrication sur l'ensemble des fromages sont restées faibles. Des valeurs d'activité leucine aminopeptidase (Lap) similaires dans les 2 cas $(142,8 \pm 41,1$ et 125,6 $\pm 36,6$ ) indiquaient un développement identique des levains sur les 2 types de fromages pendant la fabrication.

\section{Composition des fromages à 4 mois}

\section{Influence du facteur traitement du lait sur les caractéristiques physico-chimiques}

Pour cette comparaison, les 6 couples de fromages (cru et microfiltré) fabriqués à partir de laits différents et avec les 6 levains $A 1, A 2, A 4, A 5, A 7$ et $A 8$ ont été pris en compte (tableau II).

Après 4 mois d'affinage (tableau III), les fromages fabriqués avec du lait microfiltré présentaient des concentrations en lactates plus fortes et des teneurs en acide acétique et propionique plus faibles que les fromages au lait cru; ces résultats indiquaient que la fermentation propionique avait été considérablement réduite dans ces fromages à cause de l'élimination de la flore propionique par microfiltration. Aucune différence significative n'est apparue entre les 2 types de fabrication pour les analyses d'acide butyrique et caproïque.

Au niveau de la protéolyse primaire, on a observé une différence significative entre les 2 types de fabrication. Les fromages fabriqués à partir de lait microfiltré présentaient une proportion de caséine $\beta$ plus faible et une proportion de caséine $\alpha_{s 1}$ plus élevée que celles des fromages fabriqués au lait cru.

En ce qui concerne les fractions azotées, les rapports NPT/NT et NPT/NS étaient plus élevés pour les fromages fabriqués au lait cru que sur les fromages fabriqués à partir de lait microfiltré. On a noté une faible influence de la flore des laits sur le classement des associations selon les rapports 
Tableau III. Analyse de variance des mesures physico-chimiques (moyenne \pm écart type) réalisées sur fromages de 4 mois.

Analysis of variance of the physico-chemical measurements (mean \pm standard deviation) made on 12 four-month-old cheeses.

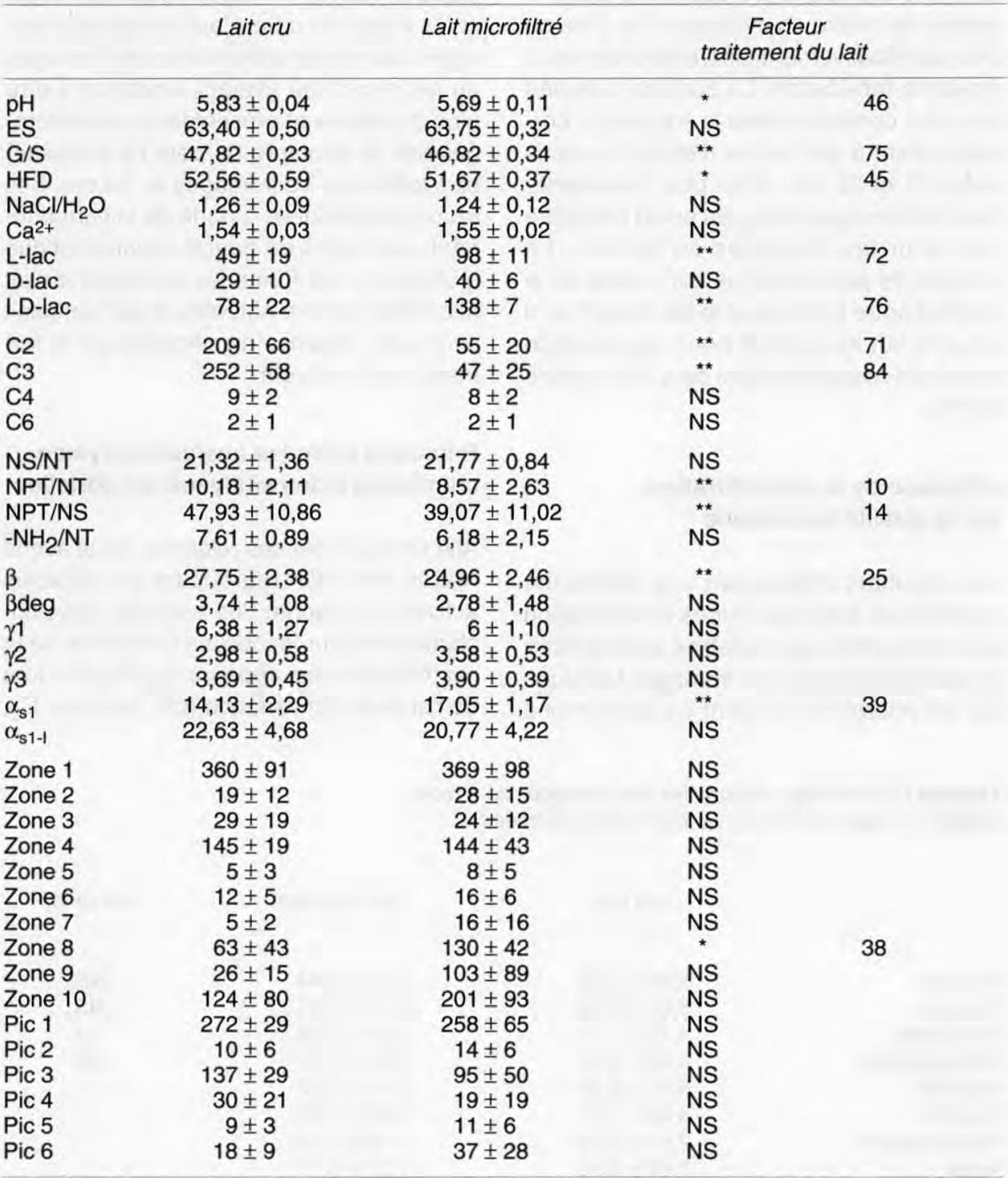

Seuil de signification : non significatif (NS), significatif au seuil de $5 \%\left({ }^{\star}\right)$, de $1 \%\left({ }^{\star \star}\right)$, de $0,1 \%\left({ }^{\star \star \star}\right)$ suivi du pourcentage de la variance expliquée par le facteur. ES : extrait sec $(\mathrm{g} / 100 \mathrm{~g}$ fromage); HFD : humidité du fromage dégraissé ( $\mathrm{g} / 100 \mathrm{~g}$ fromage dégraissé) ; G/S : gras sur sec ; $\mathrm{Ca}^{2+}$ : calcium (g/100 g ES); $\mathrm{NaCl}$ (g/100 g eau) ; L-lac, D-lac, LDlac : acide lactique $(\mathrm{mmol} / \mathrm{g}) ; \mathrm{C} 2, \mathrm{C} 3, \mathrm{C} 4, \mathrm{C} 6$ : acides gras volatils $(\mathrm{mg} / 100 \mathrm{~g})$; fractions azotées $(\mathrm{g} / 100 \mathrm{~g} \mathrm{NT})$; caséines (\% par rapport à la surface totale); "pic" et «zone" (fig 1, $10^{4} \times$ unités arbitraires) .

Significant level: non significant (NS), significant $\mathrm{P}<0.005\left(^{*}\right)$, significant $\mathrm{P}<0.01\left(^{* *}\right)$, significant $\mathrm{P}<0.001\left(^{* * *}\right)$ followed by the percent of the variance explained by the factor; ES: dry matter ( $g / 100 \mathrm{~g}$ cheese); HFD: moisture in nonfat cheese ( $g / 100 \mathrm{~g}$ non-fat cheese); G/S: fat on dry matter; Ca ${ }^{2+}$ : calcium (g/100 g ES); $\mathrm{NaCl}(\mathrm{g} / 100 \mathrm{~g}$ water); $\mathrm{L}-\mathrm{lac}$, D-lac, LD-lac: lactic acid (mmol/g); C2, C3, C4, C6: volatile fatty acids ( $\mathrm{mg} / 100 \mathrm{~g}) ;$ nitrogen fractions $(\mathrm{g} / 100 \mathrm{~g}$ of total nitrogen); caseins (\% of total surface); "pic" and "zone" (fig 1, $10^{4} x$ arbitrary units); 
azotés, soit : $A 2>A 5>A 1>A 8>A 4>A 7$ pour le lait cru et $A 2>A 5>A 8>A 1>A 7>A 4$ pour le lait microfiltré. L'analyse des profils CLHP a permis de mettre en évidence une zone de pics significativement différente entre les 2 types de fabrication. La surface cumulée des pics contenus dans la "zone 8 ", correspondant à des temps d'élution compris entre 31 et $36 \mathrm{~min}$, était plus importante pour les fromages fabriqués au lait microfiltré que pour les fromages au lait cru. Le nombre de pics contenus dans cette zone oscillait entre 8 et 9 pour le lait cru et 6 et 9 pour le lait microfiltré pour une surface moyenne respectivement de $4,90 \%$ contre $8,64 \%$.

\section{Influence de la microfiltration sur la qualité sensorielle}

Les résultats indiquaient une différence significative entre les 2 types de fabrication pour l'ensemble des variables caractérisant le goût (tableau IV). Les fromages fabriqués au lait microfiltré avaient un goût moins intense, ils étaient moins typés, plus amers mais en revanche moins acides et moins piquants que les fromages au lait cru. Même si les notes de pâte n'apparaissaient pas significativement différentes, les fromages au lait microfiltré avaient tendance à être plus élastiques et présentaient une hétérogénéité de structure de pâte (granulosité) plus faible que les fromages au lait cru. Ces fromages fabriqués à partir de lait cru donnant une meilleure qualité organoleptique globale que les fromages fabriqués au lait microfiltré, la flore naturelle du lait semblait avoir une influence significative sur la formation de la flaveur.

\section{Relations entre les analyses physico- chimiques et les analyses sensorielles}

Afin de visualiser ces relations, nous avons réalisé une ACP regroupant en variables actives, la majorité des analyses physicochimiques pour lesquelles l'influence de la microfiltration est apparue significative lors de l'analyse de variance $\left(\mathrm{pH}\right.$, lactates, $\mathrm{C}_{3}$,

Tableau IV. Analyses sensorielles des fromages de 4 mois.

Sensory measurements on 12 four-month-old cheeses.

\section{Lait cru}

$5,48 \pm 0,65$

$5,62 \pm 0,49$

$4,72 \pm 0,74$

$4,48 \pm 0,61$

$5,91 \pm 0,40$

$4,93 \pm 0,74$

$2,04 \pm 0,96$

$0,67 \pm 0,59$

$5,26 \pm 0,40$

$0,60 \pm 0,36$

$6,02 \pm 0,38$

$6,07 \pm 0,56$
Lait microfiltré

Test rangs

$6,14 \pm 0,44$

NS

$6,18 \pm 0,61$

NS

$4,64 \pm 0,59$

$3,58 \pm 1,02$

$5,14 \pm 0,32$

$3,59 \pm 0,43$

$1,19 \pm 0,39$

$1,31 \pm 0,77$

$4,92 \pm 0,27$

$1,60 \pm 1,00$

$6,08 \pm 0,31$

$4,89 \pm 0,38$
NS

NS

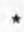

$\star$

*

*

NS

Seuil de signification : non significatif (NS), significatif au seuil de $5 \%\left({ }^{*}\right)$.

Significant level : non significant (NS), significant $\mathrm{P}<0.005\left(^{*}\right)$. 
$\alpha_{s 1}, \beta$, zone 8, NPT/NT) et en variables supplémentaires, l'ensemble des analyses sensorielles. Les variables $\mathrm{C} 2$ et $\mathrm{C} 3$ d'une part, NPT/NT et NPT/NS d'autre part étant bien corrélées entre elles (respectivement $r=$ 0,894 et $r=0,968$ ), seules ont été prises en compte dans l'ACP les variables $\mathrm{C} 3$ et NPT/NT (fig 2). Le cercle de corrélation dans le plan principal (axes 1 et 2 ) absorbait $78 \%$ de l'inertie et donnait ainsi une bonne représentation schématique des relations entre variables. On a noté que les variables de qualité : goût et typicité, de même que l'acide propionique et la teneur globale en lactates étaient mieux corrélés à l'axe 1 que le rapport NPT/NT, plutôt corrélé à l'axe 2 . Ces résultats indiquaient que la protéolyse secondaire était ici davantage liée aux caractéristiques de pâte du fromage (hétérogénéité de structure) bien que l'intensité du goût semblait évoluer dans le même sens que la proportion de caséine $\beta$. Les fromages au lait cru présentant un taux de caséine $\beta$ plus important ont en effet donné un goût plus intense que les fromages au lait microfiltré. II était intéressant de noter la corrélation entre les critères typicité, goût et les teneurs en acide propionique. On a nettement vu ici que dans le cas des fromages cuits pressés, le goût du fromage impliquait non seulement la protéolyse mais aussi la fermentation propionique.

On a noté également que la «zone 8» évoluait presque en sens opposé à l'intensité du goût. Les fromages fabriqués au lait microfiltré ayant donné une zone 8 importante étaient des fromages ayant peu de goüt $(4,89$ contre 6,07 en lait cru). Les corrélations positive et négative trouvées respectivement entre le rapport NPT/NS et la zone $1(r=0,717)$ et le rapport NPT/NS et la zone $8(r=-0,671)$ suggéraient que les pics de la zone 1 correspondaient plutôt à des acides aminés ou peptides de petite taille alors que ceux de la zone 8 correspondaient davantage à des peptides de taille plus grande. Ainsi, plus on aurait de peptides peu dégradés dans la zone 8 , plus l'intensité du goût serait faible.

La projection du nuage de points sur un plan a montré que les fromages se répartissaient selon 2 axes décrits par les variables C3 (axe 1) et NPT/NT (axe 2). Même si le fromage fabriqué au lait cru avec l'association A1 était mal représenté dans ce plan, les autres fromages fabriqués au lait cru donnaient des valeurs de C3 élevées, ainsi que de bonnes notes globales de goût, tandis qu'à l'opposé, les fromages fabriqués au lait microfiltré se répartissaient selon un axe décrit par la variable NPT/NT qui paraissait très liée à la nature du levain. Par ailleurs, en reliant 2 à 2 les fromages fabriqués à partir du même levain, on visualisait nettement l'amélioration du goût et de la typicité des fromages lorsqu'on passait du lait microfiltré au lait cru. On a remarqué que chaque levain se définissait, quel que soit le lait, par un niveau de protéolyse, sauf exceptions pour les levains $A 3$ et $A 9$ (moins protéolytiques sur lait cru) et dans une moindre mesure $A 4$ et $A 7$ (où la tendance est inversée). On a noté également qu'il n'existait pas toujours de corrélation entre les tests de laboratoire et les mesures de protéolyse réalisées sur fromages. Ainsi, le levain A3 dont les souches présentaient individuellement une plus grande activité protéolytique que celles composant le levain A2 n'exprimait pas ce caractère dans le fromage fabriqué à partir de lait microfiltré puisque ces 2 associations avaient un positionnement voisin dans la représentation ACP. Comme nous l'avions déjà vu dans une étude précédente (Bouton et al, 1993), ce résultat pouvait s'expliquer par des conditions expérimentales différentes telles que le substrat lait : il ètait possible que la capacité de synthèse des peptidases du levain A2 fut améliorée lorsque ce mélange était cultivé sur lait plutôt que sur milieu synthétique. Par ailleurs, si le substrat lait était différent, les conditions de température choisies pouvaient influencer la lyse cellulaire et donc la protéolyse. 

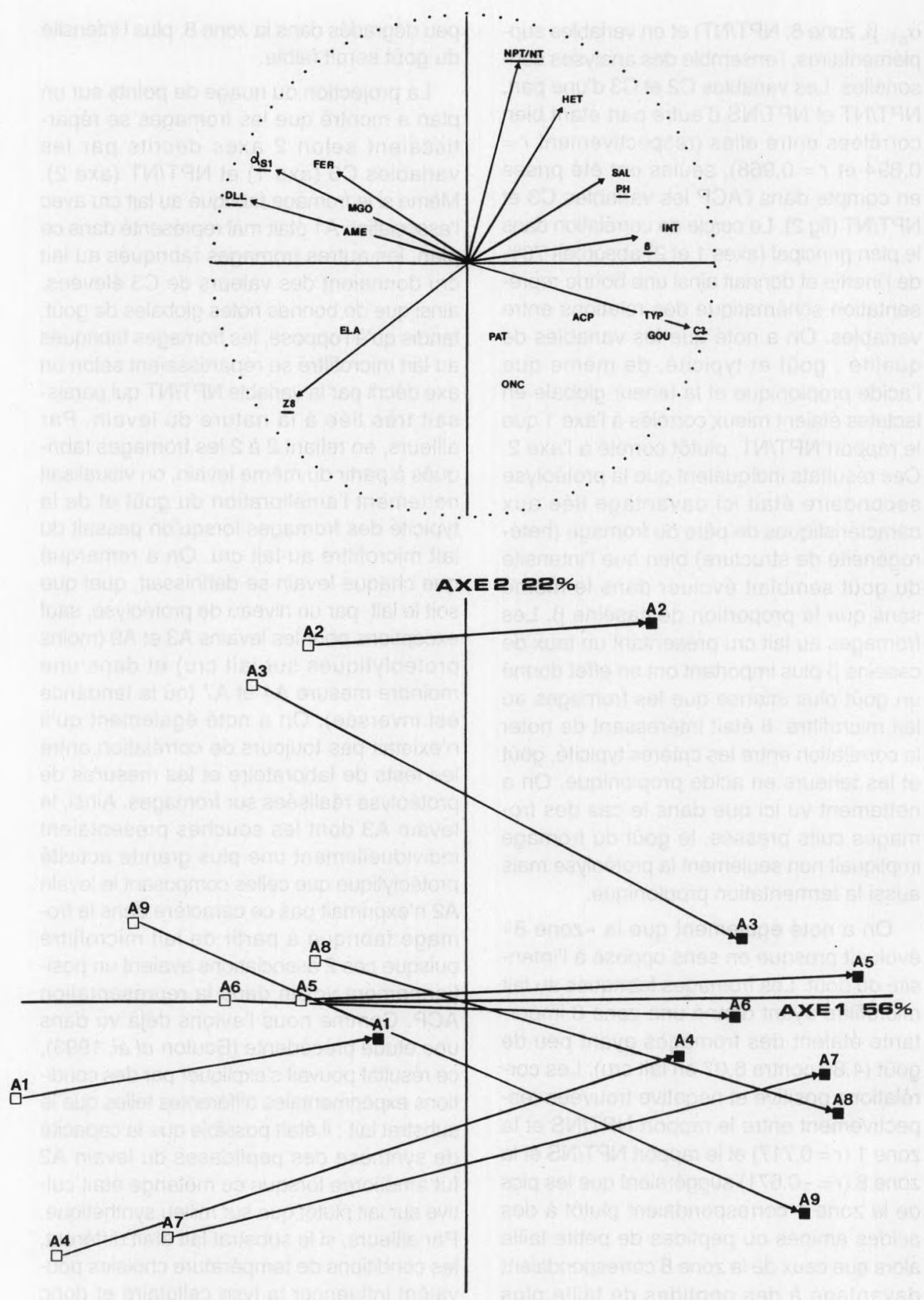


\section{DISCUSSION}

Au cours de l'affinage du gruyère, les bactéries lactiques thermophiles disparaissent progressivement : les lactobacilles ne représentent plus en fin d'affinage que $0,1 \%$ de la population atteinte pendant la fabrication, tandis que Streptococcus salivarius subsp thermophilus n'est plus détectable (Veaux et al, 1974). Toutefois, d'autres micro-organismes se développent, tels que les bactéries propioniques responsables de la fermentation du lactate et de la formation de l'ouverture. Ainsi, dans les fromages de type emmental, fabriqué à partir de lait thermisé, la population de bactéries propioniques atteint $10^{8} \mathrm{UFC} / \mathrm{g}$ après 2 semaines d'affinage en cave chaude et $10^{9}$ UFC/g en fin d'affinage en cave chaude (Thierry et al, 1994). Ces bactéries (Propionibacterium arabinosum, $P$ pentosaceum, $P$ shermanii) possèdent un large éventail de peptidases actives jusqu'à pH 5,0 et pourraient donc participer à la dégradation des protéines (Floberghagen et al, 1978 ; Sahlström et al, 1989). Cela contribuerait à expliquer les résultats de protéolyse plus élevés obtenus avec certaines associations en lait cru (augmentation par exemple du rapport NPT/NT avec l'association A2, A4 et A7).

Toutefois, dans certains cas nous avons observé une activité protéolytique plus faible (diminution du rapport NPT/NT avec l'association $\mathrm{A} 3$ et $\mathrm{A} 9$ ). On sait que si la capacité de développement et de fermentation des bactéries propioniques peut être stimulée par d'autres micro-organismes, elle peut également être inhibée ; ainsi, sur 12 souches de lactobacilles testées, 2 souches de $L$ helveticus, 2 souches de $L$ delbrueckii subsp lactis et une souche de $L$ acidophilus inhibent la croissance des bactéries propioniques (Winkler, 1953). Plus récemment Perez Chaia et al (1994) ont mis en évidence l'inhibition de $P$ acidipropionici par $L$ helveticus. Cependant, les fromages au lait cru issus des associations $A 3$ et $A 9$ présentent des taux d'acide propionique (respectivement 260 et $307 \mathrm{mg} / 100 \mathrm{~g}$ ) plus élevés que sur lait microfiltré ( 10 et $13 \mathrm{mg} / 100$ g) et proches de la valeur moyenne de l'ensemble des 18 fromages fabriqués au lait cru $(259 \mathrm{mg} / 100 \mathrm{~g})$; ces résultats indiquent que les bactéries propioniques se sont développées correctement. La diminution de la protéolyse dans ces fromages au lait cru est peut être due à un développement plus faible des souches composant les levains A3 et A9. Des compétitions plus ou moins importantes pour les acides aminés et les petits peptides ont pu intervenir entre la flore naturelle du lait cru et la flore des levains. D'autre part, certains microorganismes telles que les bactéries lactiques présentes dans la flore indigène du lait sont capables de produire des bactériocines pouvant inhiber le développement d'autres bactéries lactiques issus des levains (Piard et Desmazeaud, 1992 ; Barefoot et Nettles, 1993).

Fig 2. Analyse en composantes principales des analyses physico-chimiques et sensorielles. Fromages fabriqués au lait cru (घ) et microfiltré $(\square)$; ONC : onctuosité ; PAT : pâte ; ACP : aciditépiquant ; GOU : goût ; TYP : typicité ; INT : intensité du goût ; SAL : salé ; HET : hétérogénéité ; FER : fermeté ; MGO : mauvais goût ; AME : amertume ; ELA : élasticité ; $C 3$ : acide propionique ; DLL : acide lactique $; \alpha_{s 1}, \beta$ : caséine $\alpha_{s 1}, \beta ;$ NPT/NT : fraction azotée $; z 8$ : "zone" 8 . Seules les variables actives sont soulignées.

Principal component analysis of physico-chemical and sensory measurements. Cheeses made from raw (घ) and microfiltered (এ) milk; ONC: unctuousness ; PAT: paste; ACP: acidity-pungent; GOU: taste; TYP: typicity; INT: taste intensity; SAL: salty; HET: heterogeneity; FER: firmness; MGO: bad taste; AME: bitterness; ELA: springiness; C3: propionic acid; DLL: lactic acid; $\alpha_{s 1}, \beta: \alpha_{s 1}, \beta$ casein ; NPT/NT: nitrogen fraction ; 28 : "zone" 8 . Only active variables are underlined. 
Par ailleurs, d'autres bactéries mésophiles se développent au cours de l'affinage du gruyère, constituant la flore dominante ( $10^{8}$ à $10^{9}$ bactéries/g de fromage). Il s'agit principalement de $L$ casei var casei et des bactéries du genre Pediococcus. En flore sous-dominante ( $10^{6}$ à $10^{8}$ bactéries $/ g$ ), on trouve $L$ casei var rhamnosus, $L$ fermenti, $L$ buchneri et des streptocoques du groupe $\mathrm{D}$ (Veaux et al, 1974). D'après Tittsler et al (1948), les lactobacilles homofermentaires facultatifs tels $L$ casei et $L$ plantarum améliorent généralement la flaveur du cheddar, tandis que les lactobacilles hétérofermentaires obligatoires tels $L$ brevis et $L$ fermenti produisent généralement des flaveurs indésirables.

Par ailleurs, Martley et Crow (1993) ont mis en évidence sur des fabrications de type emmental (Swiss cheese) réalisées en présence de $L$ plantarum, une diminution des fermentations lactique et propionique ; ainsi, certaines souches résistantes à des températures de $52^{\circ} \mathrm{C}$, utilisant les citrates sont capables d'interférer avec l'activité des bactéries propioniques et donc d'affecter la formation des yeux et le développement de la flaveur des fromages. Comme nous avons pu le voir sur le comté, Mc Sweeney et al (1993) ont également observé sur le cheddar que les fromages fabriqués au lait cru contenaient plus d'acides aminés libres, en particulier plus d'acide glutamique que les fromages fabriqués à partir de lait microfiltré ; à 3 mois d'affinage, ces 2 types de fabrication présentaient des différences de profils peptidiques. L'auteur suggère que cette protéolyse plus importante dans le cheddar au lait cru résulte d'une activité peptidasique et protéasique plus élevée, provenant certainement des lactobacilles hétérofermentaires facultatifs appartenant aux espèces de $L$ casei et $L$ plantarum. Grâce à leur équipement enzymatique de type protéolytique et lipolytique, ces bactéries peuvent donc également participer aux différents phénomènes de l'affinage (Khalid et Marth, 1990). On peut donc regretter qu'une étude bactériologique des fromages affinés n'ait pas été effectuée ce qui aurait permis de préciser le rôle de certains groupes bactériens dans la protéolyse et la flaveur des fromages.

D'autre part, nous avons vu que l'intensité du goût évolue dans le même sens que la proportion de caséine $\beta$. En effet, les fromages au lait microfiltré présentent une plus faible proportion de caséine $\beta$ que les fromages au lait cru. Cette plus faible teneur en caséine $\beta$ peut être due à un appauvrissement du lait en caséine $\beta$ par microfiltration ou à une activation du complexe plasmineplasminogène par inactivation de l'inhibiteur de l'activateur du plasminogène (Fox, 1992) liée au chauffage de la crème $\left(85^{\circ} \mathrm{C}\right.$, $30 \mathrm{~s}$ ). II serait donc intéressant de suivre l'évolution de cette caséine $\beta$ et de ses produits de dégradation au cours du temps pour chaque type de lait afin de déterminer s'il existe une réelle activation de la plasmine ou bien si une fraction des micelles de caséine $\beta$ est retenue par la membrane au cours de la microfiltration.

Si le rôle des bactéries lactiques des levains dans la protéolyse et par là même dans la flaveur des fromages à pâte pressée cuite n'est plus à démontrer, on s'aperçoit que les micro-organismes du lait interviennent de façon non négligeable dans ces mécanismes. Cette étude montre clairement le rôle de ces «micro-organismes non levains" dans la protéolyse et la flaveur, puisque les fromages fabriqués à partir de lait cru étaient plus protéolysés et plus typés que les fromages fabriqués à partir de lait microfiltré. D'après Gallmann (1982), il apparaît que si chaque micro-organisme peut être tenu pour partiellement responsable des changements typiques constatés dans le fromage "cru", aucune des souches considérées n'est capable à elle seule de produire son goût spécifique. II faut plutôt admettre que seule l'action conjuguée de toute la flore microbienne du lait cru permet 
de reproduire les caractéristiques du fromage à base de lait cru. Une meilleure connaissance de cette flore et des phénomènes d'interactions existant entre cette flore indigène du lait et les bactéries du levain mérite donc d'être développée, afin de pouvoir choisir au mieux les souches constituant les levains, en fonction du site de fabrication.

\section{REMERCIEMENTS}

Nous remercions G Duboz de l'INRA de Poligny pour la réalisation des fabrications fromagères, $M$ $H$ Duployer et $P$ Guyot pour leur contribution aux analyses physico-chimiques et microbiologiques, $S$ Pochet et $C$ Borde pour leur assistance technique lors de la réalisation des dosages chromatographiques ainsi que tous les membres du jury d'analyse sensorielle.

Ce programme a reçu le soutien financier du Comité interprofessionnel du gruyère de Comté et de l'Association pour le développement de la recherche dans l'industrie laitière (Arilait, Paris).

\section{RÉFÉRENCES}

Anonyme (1985) Lait, détermination de la teneur en protéines, méthode au noir amido. FIL 98A, Bruxelles

Anonyme (1990) Lait. Détermination de la teneur en matière grasse, méthode acido-butyrométrique. NF V04-210 AFNOR, Paris

Anonyme (1991) Lait et produits laitiers. Dénombrement des micro-organismes. Comptage des colonies à $30^{\circ} \mathrm{C}$. FIL 100B, Bruxelles

Barefoot SF, Nettles CG (1993) Antibiosis revisited: bacteriocins produced by dairy starter cultures. J Dainy Sci 76, 2366-2379

Bertozzi L, Panari G (1993) Cheeses with appellation d'origine contrôlée $(A O C)$ : factors that affect quality. Int Dairy J3, 297-312

Bouton $Y$ (1992) Caractérisation approfondie sur minifromages de l'activité protéolytique de souches et associations de souches de bactéries lactiques thermophiles utilisées en fabrication de Comté. Relation avec les caractéristiques organoleptiques. Thèse de Doctorat, Nancy I, France

Bouton Y, Guyot P, Dasen A, Grappin R (1993) Activitè protéolytique de souches de lactobacilles thermophiles isolées de levains et de Comté. I. Validation sur minifromages des techniques de laboratoire. Lait 73, 265-279

Bouton Y, Guyot P, Dasen A, Grappin R (1994) Activité protéolytique de souches de lactobacilles thermophiles isolées de levains et de Comté. II. Applications en sites industriels. Lait 74, 33-46

Bouton Y, Gappin R (1994) Measurement of proteolysis in cheese : relationship between phosphotungstic acid-soluble $\mathrm{N}$ fraction by Kjeldahl and 2,4,6 trinitrobenzene-sulphonic acid reactive groups in watersoluble N. J Dairy Res 61, 437-440

Floberghagen V, Sorhaug T, Langsrud T (1978) Peptides hydrolases des bactéries propioniques. Brèves Commun 20e Congr Int Lait, Paris, 483-484

Fox PF (1992) Indigenous enzymes in milk. III. Proteinases. In: Advanced dairy chemistry vol 1 Proteins (P F Fox ed), Elsevier Applied Science, London

Gallmann P (1982) Einfluss der Rohmilchflora auf die biochemischen Vorgănge der Kăsereifung am Beispiel von Raclette-Kăse aus pasteurisierter und roher Milch. These von Eigenössischen Technischen Hochschule n ${ }^{\circ} 6972$, Zürich

Gripon JC, Desmazeaud MJ, Le Bars D, Bergère JL (1975) Étude du rôle des micro-organismes et des enzymes au cours de la maturation. II. Influence de la présure commerciale. Lait 55, 502-516

Jaubert G, Costes P, Guyonnet P, Gay MF, Pierre A, Maubois JL (1991) Une épuration bactérienne à presque $100 \%$. Process 1066, 62-67

Khalid NM, Marth EH (1990) Lactobacilli. Their enzymes and role in ripening and spoilage of cheese : a review. J Dairy Sci 73, 2669-2684

Kuchroo CN, Fox PF (1982) Soluble nitrogen in Cheddar cheese : comparison of extraction procedures. Milchwissenschaft 37, 331-335

Martley FG, Crow VL (1993) Interactions between non starter microorganisms during cheese manufacture and ripening. Int Dairy $J 3,461-483$

Mc Sweeney PL H, Fox PF, Lucey JA, Jordan KN, Cogan TM (1993) Contribution of the indigenous microflora to the maturation of Cheddar Cheese. Int Dairy J 3, 613-634

Perez Chaia A, Strasser de Saad AM, Pesce de Ruiz Holgado A, Oliver G (1994) Competitive inhibition of Propionibacterium acidipropionici by mixed culturing with Lactobacillus helveticus. J Food Prot 57. 341-344

Piard JC, Desmazeaud M (1992) Inhibiting factors produced by lactic acid bacteria. 2. Bacteriocins and other antibacterial substances. Lait 72, 113-142

Sahlström S, Espinosa C, Langsrud T, Sorhaug T (1989) Cell wall, membrane and intracellular peptidase activities of Propionibacterium shermanii. J Dairy Sci $72,342-350$

Thierry A, Madec MN, Richoux R (1994) Croissance des bactéries propioniques dans le fromage : com- 
paraison de 2 milieux de dénombrement. Lait 74, 161-171

Tittsler RP, Sanders GP, Lochry HR, Sager OS (1948) The influence of various lactobacilli and certain streptococci on the chemical changes, flavor development and quality of Cheddar cheese. J Dairy Sci 31, 716

Trouvé E, Maubois JL, Piot M et al (1991) Rétention de différentes espèces microbiennes lors de l'épuration du lait par microfiltration en flux tangentiel. Lait 71, 1-13

Veaux M, Accolas JP, Vassal L, Auclair J (1974) Évolution des bactéries lactiques et des bactéries pro- pioniques au cours de la fabrication et de l'affinage du gruyère. Brèves Commun $19^{\circ}$ Congr Int Lait, New Delhi, 1F, 458-459

Vincens D, Tabard J (1988) L'élimination des germes bactériens sur membranes de microfiltration. Procédé Alfa-Laval. Tech Lait Market 1033, 62-64

Wilcoxon $F$ (1945) Individual comparisons by ranking methods. Biometrics 1, 80-83

Winkler S (1953) The inhibitory action of propionic acid bacteria. 13e Int Dairy Congr The Hague, 3, 11641167 